\title{
Image segmentation and analysis for densification mapping of nanoporous gold after nanoindentation
}

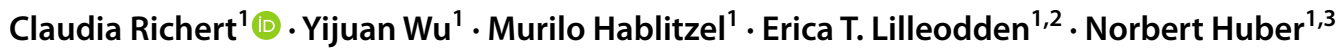

Received: 2 April 2021 / Accepted: 27 June 2021 / Published online: 14 July 2021

(c) The Author(s) 2021

\begin{abstract}
Segmentation of scanning electron microscopy (SEM) images of focused ion beam (FIB) cross-sections through indented regions in nanoporous gold (np-Au) is carried out. A key challenge for image analysis of open porous materials is the appropriate binarization of the pore and gold ligament regions while excluding material lying below the cross-sectional plane. Here, a manual approach to thresholding is compared to global and local approaches. The global thresholding resulted in excessive deviations from the nominal solid fraction, due to a strong gray-scale gradient caused by the tilt angle during imaging and material shadowing. In contrast, the local thresholding approach delivered local solid fractions that were free of global gradients, and delivered a quality comparable to the manual segmentation. The extracted densification profiles vertically below the indenter as well as parallel to the surface showed an exponential-type decay from the indenter tip towards the nominal value of 1 far from the indenter.
\end{abstract}

\section{Introduction}

Nanoporous gold (np-Au) is an ideal model material for fundamental research on the structure-property relationships of open pore materials. An overview of the fascinating morphologies and mechanical properties of this material is provided in a review article [1]. While macroscopic compression testing assumes a homogeneous deformation of representative volumes within the sample, nanoindentation allows studying the densification behavior under heterogeneous strains across heterogeneous volumes. While such studies provide useful insight into the deviation from the nanoindentation strain field in monolithic, fully dense materials, quantified image analyses are needed-a non-trivial

Norbert Huber was an editor of this journal during the review and decision stage. For the MRS Advances policy on review and publication of manuscripts authored by editors, please refer to mrs. org/editor-manuscripts.

Claudia Richert

claudia.richert@hereon.de

1 Institute of Materials Mechanics, Helmholtz-Zentrum Hereon, Geesthacht, Germany

2 Institute of Advanced Ceramics, Hamburg University of Technology, Hamburg, Germany

3 Institute of Materials Physics and Technology, Hamburg University of Technology, Hamburg, Germany undertaking in open-porous materials. Nonetheless, various studies have been reported, e.g. [2, 3]. Leitner et al. considered the effect of heat treatment on the densification of np-Au [2], while Briot et al. prepared a cross-section of a np-Au sample after nanoindentation and investigated the densification as function of the depth [3] via SEM analysis. In the latter study, the densified region is split into seven parabolic segments that were aligned to the outer perimeter of the densified region. Relative to the nominal solid fraction, the data showed an increased value with a peak value in zone 4, which is about the half distance to the contact radius; at the outermost segment the analysis delivered a densification of $\sim 30 \%$.

Due to the challenges that come along with the high depth of field achievable by SEM imaging, sophisticated image processing routines have been reported for the analysis of nano-structured materials in e.g. [4-7]. Such workflows contain sequential steps of image acquisition, pre-processing, segmentation, post-processing and validation [5], followed by the quantitative analysis [6]. The pre-processing can include the tasks of noise reduction, contrast enhancement and illumination correction, as summarized in [5]. For the choice of the segmentation approach, ranging from manual to automated custom-designed, the image characteristics and further the specific aim of the subsequent analysis should be considered [7]. 
A special challenge with the segmentation of open-pore materials is that secondary electrons from the underlying gold ligaments (i.e., below the cross-sectional cut) make a clear identification of the 2D distribution of gold difficult. Depending on the chosen greyscale threshold value and algorithm, an increased solid fraction often results. Our work sheds some light on the challenges with the segmentation of SEM images of FIB cross-sectioned np-Au after indentation and proposes an improved method for deriving the densification map of the indented region.

\section{Materials and methods}

Nanoporous gold (np-Au) samples were fabricated from a $\mathrm{Au}_{25} \mathrm{Ag}_{75}$ master alloy by electrochemical dealloying. Details regarding the dealloying can be found elsewhere [8]. Finally, the sample was coarsened by annealing at 500 ${ }^{\circ} \mathrm{C}$ for $1 \mathrm{~h}$ in air, resulting in a nominal ligament diameter of $300 \mathrm{~nm}$, as measured by SEM inspection. Nanoindentation testing was carried out on the polished surface of the sample using a Nanoindenter (Nano XP, MTS Corp.) equipped with a diamond Berkovich tip. Indentations were carried out at a constant strain rate of $0.05 / \mathrm{s}$ to a displacement of $20 \mu \mathrm{m}$, and then holding the load for $10 \mathrm{~s}$ followed by unloading. The average of elastic modulus and hardness was $130 \mathrm{MPa}$ and $6 \mathrm{MPa}$, respectively. Cross-sectional cuts were then made perpendicular to the indented surface through the center of the indent using focused ion beam (FIB) milling. SEM imaging and FIB milling were performed using a dual-beam system (Nova Nanolab 200, FEI Corp.) equipped with a platinum (Pt) gas injection system. Depositing a Pt layer on the indentation prevented the subsequent exposure of the analyzed surface to the $\mathrm{Ga}+$ ion beams, allowing a better crosssectional view at the surface. The FIB milling took place in two steps: first the "regular cross-section" milling protocol was employed, followed by the "cleaning cross-section" protocol, both with a current of $1 \mathrm{nA}$ and excitation voltage of $30 \mathrm{kV}$. For SEM imaging, a low voltage of $5 \mathrm{kV}$ was used, with a current of $1.6 \mathrm{nA}$. An in-lens backscattered electron detector was used, with a low voltage $(5 \mathrm{kV})$ to maximize visibility and to ensure appropriate contrast at the edge of the ligaments. Figure 1 shows an SEM micrograph taken at an angle of $52^{\circ}$ relative to the perpendicular cross-section of an indent. The dashed lines indicate the edges between the faces of the indenter. The inset shows a sketch of the relative positioning of the Pt deposition and the cross-section relative to the indenter triangular in-plane contact. The cross-section was terminated at the mid-section of the indent, as evidenced by the visibility of the sharp apex.

\section{Results and discussion}

For the image analysis, the 8-bit greyscale image needs to be binarized, i.e. converted into black and white pixels. The most common method is the global threshold value $[2,3]$. This threshold is set to a greyscale-value between 0 (black) and 255 (white). For np-Au, the initial solid fraction $\varphi=0.3 \pm 0.02$ of the sample as global value is known from pycnometry. Under the assumption that the porosity is distributed evenly within the sample, the threshold can be tuned using the image greyscale histogram. For the SEM images shown in Fig. 1a, the usual approach of applying a global threshold was found to be not suitable. As observed in Fig. 1b, due to the tilted imaging configuration, as discussed in [4], and-most importantly - the surrounding material of the cross-sectional trench, a strong gradient in grayscale results.

The applied workflow consists of a minimum number of image processing steps. To remove the global gradient, a local threshold approach was applied. An individual threshold value is calculated for each pixel, depending on the distribution of greyscale-values in just the surrounding (local)

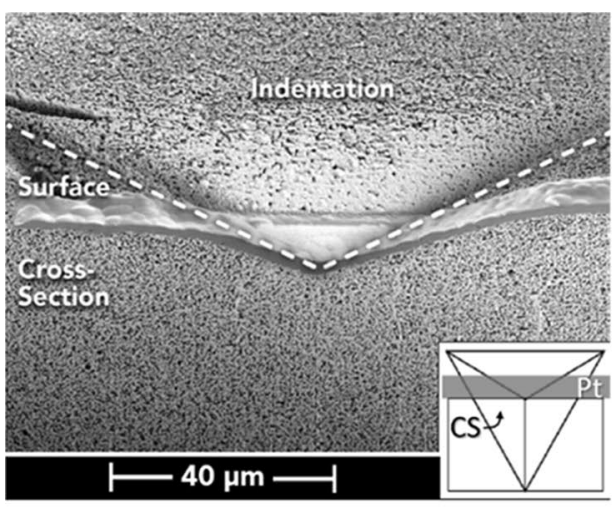

a

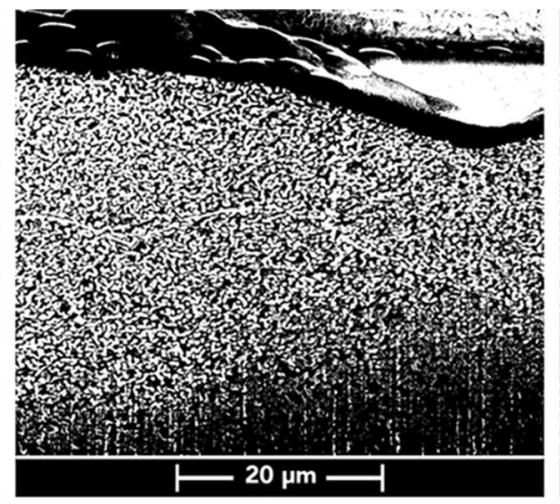

$\mathrm{b}$

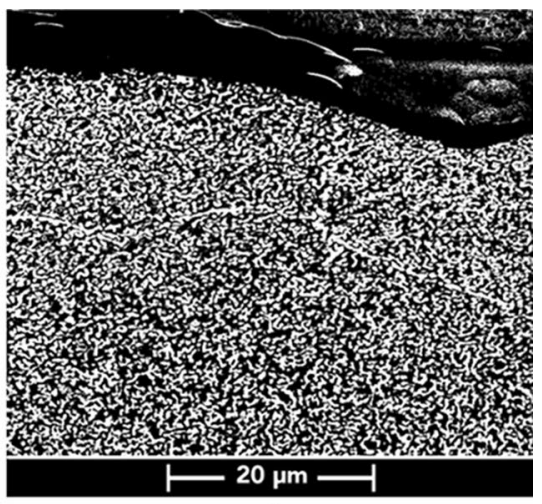

c

Fig. 1 a SEM micrograph of the cross-section analyzed in this study, b binarized image with a global grey-scale value of 183, and $\mathbf{c}$ binarized image using the Niblack algorithm in a local threshold approach with a local region of radius $r=15$. Images $\mathbf{b}$ and $\mathbf{c}$ are magnifications from $\mathbf{a}$ 
circular region. Details about global and local segmentation methods considering porous materials can be found in [9]. Here the Niblack algorithm [10] implemented in ImageJ [11] was used. The Niblack algorithm calculates the threshold $T(x, y)$ for a pixel at $(x, y)$ by calculating the mean $m$ and standard deviation $s$ of the greyscale-values in its neighborhood of radius $r$ as follows: $T(x, y)=k \cdot s(r)+m(r)$ [10]. The resulting image shows a uniform black and white field, see Fig. 1c. However, as a result of the local thresholding, the solid fraction far away from the indentation is much higher $(52 \%)$ than that of the initial sample (30\%). The question arises, whether this approach obfuscates the local densification in the area of interest, i.e., below the indented surface.

We used the time-consuming approach of manually thresholding the image to provide a reference image for segmentation validation. Certain features are readily visible to the eye as belonging to the cross-section in the FIB cutplane and in contrast to those below the surface, so-called 'shine-through' artifacts [12], see Fig. 2a. As manually segmenting several images is excessively time consuming, machine learning approaches could be suitable. A recent study on the application of machine-learning for FIB-SEM image segmentation of porous polymer films can be found [13]. However, many data sets for the training step would be needed to this end; reliable information about both the original image and the target segmentation are critical to accurate machine learning. Therefore, our aim is to find a method that allows translating the locally binarized image into one that is at least sufficiently close to the manually binarized image. Using GIMP [14], the features lying in the FIB cut-plane were colored in white (255) pixel-by-pixel, Fig. 2b, followed by thresholding the image with this value, Fig. $2 c$ results. For the coloring, the bright edges of the cut ligament were helpful. However, these edge effects likely lead to an overestimation of the white regions by $\sim 1$ pixel. Again, the solid fraction far away from the indenter is evaluated as $44 \%-\mathrm{a}$ value greater than the expected far-field density of $\sim 30 \%$.

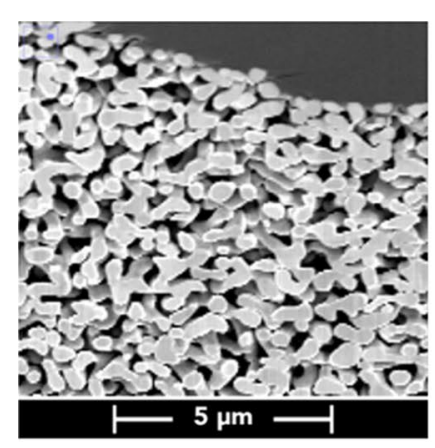

a

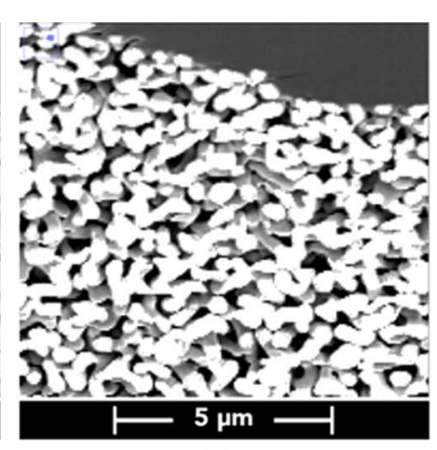

b

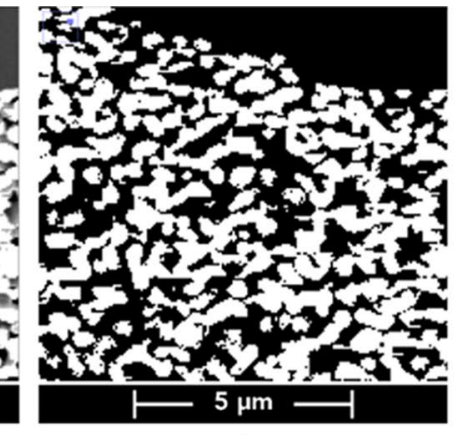

c
In comparison, the result in Fig. $2 \mathrm{~d}$ demonstrates the quality of the local thresholding with the Niblack algorithm. Although there are some small differences inside certain features, the overall agreement between manual and local binarization is good. The solid fraction evaluated in the selected sample region is $59 \%$ and $53 \%$, respectively.

Based on this result, a local densification map can be derived. After binarization with the local Niblack thresholding approach, the image is scanned in 1-pixel steps in $x$ - and $y$-directions with a chosen box size following the scan procedure by Leitner et al. [2]. At each box position, the average solid fraction $\bar{\varphi}$ in that box is calculated and attributed to the center pixel of the current scan box. The boxes of subsequent scan steps overlap, which results in a more reliable floating average of the solid fraction. To calculate the local densification $\varphi^{*}=\bar{\varphi} / \varphi_{\infty}$, the local solid fraction is normalized to the solid fraction $\varphi_{\infty}$, which is extracted from a representative region far away from the indenter tip and the surface. While due to the edge effect $\bar{\varphi}$ and $\varphi_{\infty}$ can deviate from the true solid fraction, the local densification $\varphi^{*}$ should not deviate too much and, moreover, has a consistent reference of 1 for the solid fraction that corresponds to the undeformed material. It was found, that the densification maps of the manually binarized image and the locally binarized image using Niblack algorithm qualitatively agree well.

Using this approach, the sample imaged at magnification $\times 2500$ is analyzed. The size of the image is $1024 \times 943$ pixels and the pixel edge length is approximately $100 \mathrm{~nm}$. For the binarization, the Niblack algorithm with a local neighborhood region of radius 15 pixels is applied. For the subsequent scan of the local solid fraction, a scan box size of $50 \times 50$ pixels was used. The resulting densification $\varphi^{*}$ is shown in Fig. 3a as an overlay with the binarized SEM image, where the white box in the upper left corner is the scan box, and the black frame on the right side indicates the region for measurement of the reference value $\varphi_{\infty}$. From this map, densification scans along the surface and vertically below the indenter tip can be extracted, as shown in
Fig. 2 Image processing of a selected region beneath the indenter tip: a original greyscale image showing 'shine-through' artifacts, b manual coloring of all pixels in the FIB 2D cut-plane via GIMP, $\mathbf{c}$ thresh- olding of image $\mathbf{b}$ with greyscale value 255 , and $\mathbf{d}$ result with local thresholding approach using Niblack algorithm $(r=15$ pixel $)$

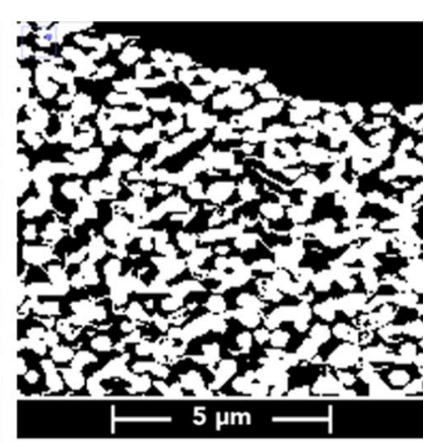

$\mathrm{d}$ 


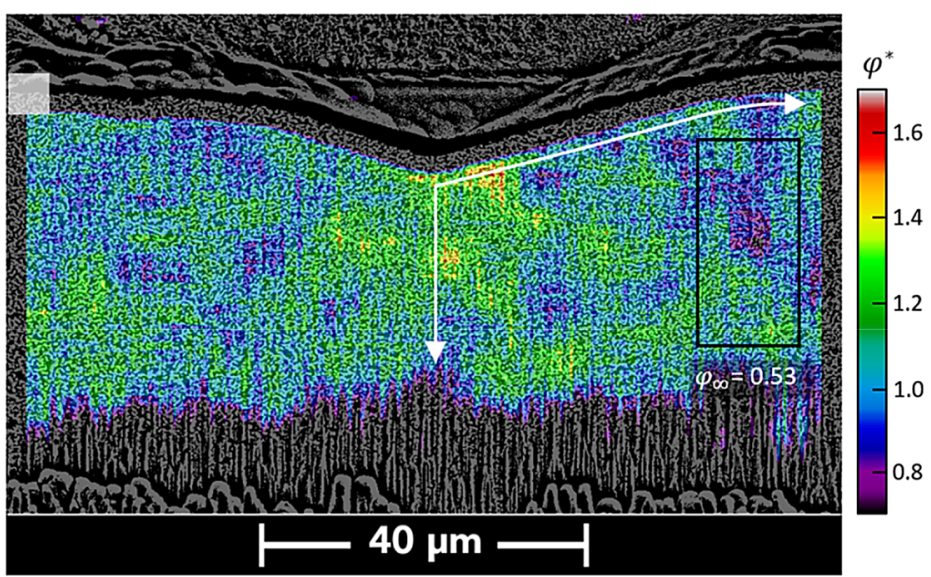

a

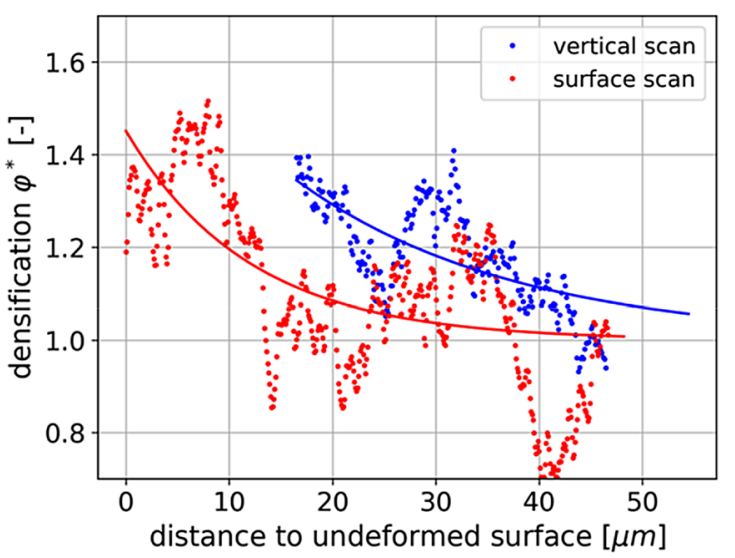

b

Fig. 3 a Binarized SEM image of sample with $300 \mathrm{~nm}$ average ligament diameter as an image overlay with the computed densification $\varphi^{*}$ color map, and $\mathbf{b}$ computed densification scan along surface and vertically below indenter tip; fit by exponential decay

Fig. 3b. Although the scans still show significant fluctuations, an overall trend is observed that follows an exponential decay function with increasing distance from the tip with an asymptotic value of 1 .

\section{Conclusions}

Image analysis of FIB-sectioned np-Au samples after nanoindentation revealed that a global thresholding can lead to considerable deviations and misinterpretations of the local solid fraction due to greyscale gradients within the image, caused be the image tilt angle or shadowing due to surrounding material outside of the trench milled in order to view the cross-section. After manual segmentation of a reference image, we showed that the local thresholding with the Niblack algorithm provides a reasonable qualification that removes the global gradient and conserves local densification introduced in the sample by nanoindentation. The extracted densification profiles show an overall increasing densification towards the indenter tip that follows an exponential function, while local maxima can be attributed to inhomogeneities within the sample. Further analyses of the resultant densification distributions are ongoing.

As an outlook considering recent literature, the segmentation workflow could be extended towards the more comprehensive workflows as proposed for images of porous structures [4-7]. Possible pre-processing tasks, such as image enhancement techniques including denoising (e.g. intensity gradient noise reduction), contrast enhancement, illumination correction [5] could be tried. An application of edge detection methods [5] appears to be not effective due to ligaments that disappear into the background. For the densification scan, a quantitative analysis of the autocorrelation length through fast Fourier transform could be tried, as used in [6]. Such techniques could provide an alternative or extension to a local threshold approach for the image segmentation of FIB cross-sections of nanoporous gold after nanoindentation.

Acknowledgments This work was supported by the Deutsche Forschungsgemeinschaft (DFG, German Research Foundation): Project Number 192346071: SFB 986 "Tailor-Made Multi-Scale Materials Systems: M3," Project B4.

Funding Open Access funding enabled and organized by Projekt DEAL.

Data availability The datasets generated and analyzed during the current study are available from the corresponding author on reasonable request.

\section{Declarations}

Conflict of interest The authors declare no conflict of interest.

Open Access This article is licensed under a Creative Commons Attribution 4.0 International License, which permits use, sharing, adaptation, distribution and reproduction in any medium or format, as long as you give appropriate credit to the original author(s) and the source, provide a link to the Creative Commons licence, and indicate if changes were made. The images or other third party material in this article are included in the article's Creative Commons licence, unless indicated otherwise in a credit line to the material. If material is not included in the article's Creative Commons licence and your intended use is not permitted by statutory regulation or exceeds the permitted use, you will need to obtain permission directly from the copyright holder. To view a copy of this licence, visit http://creativecommons.org/licenses/by/4.0/. 


\section{References}

1. J. Weissmüller, K. Sieradzki, Dealloyed nanoporous materials with interface-controlled behavior. MRS Bull. 43(1), 14 (2018)

2. A. Leitner, V. Maier-Kiener, J. Jeong, M.D. Abad, P. Hosemann, S.H. Oh, D. Kiener, Interface dominated mechanical properties of ultra-fine grained and nanoporous $\mathrm{Au}$ at elevated temperatures. Acta Mater. 121(9), 104 (2016)

3. N.J. Briot, T.J. Balk, Focused ion beam characterization of deformation resulting from nanoindentation of nanoporous gold. MRS Commun. 8(01), 132 (2018)

4. D. Kim, S. Lee, W. Hong, H. Lee, S. Jeon, S. Han, J. Nam, Image segmentation for FIB-SEM serial sectioning of a Si/C-graphite composite anode microstructure based on preprocessing and global thresholding. Microsc. Microanal. 25(5), 1139 (2019)

5. A. Iskakov, S.R. Kalidindi, A framework for the systematic design of segmentation workflows. Integr. Mater. Manuf. Innov. 9(1), 70 (2020)

6. C. Zhao, Y. Qiao, Characterization of nanoporous structures: from three dimensions to two dimensions. Nanoscale 8(40), 17658 (2016)

7. W.-T. Tsai, A. Hassan, P. Sarkar, J. Correa, Z. Metlagel, D.M. Jorgens, M. Auer, From voxels to knowledge: a practical guide to the segmentation of complex electron microscopy 3D-data. J. Vis. Exp. 90, e51673 (2014)
8. Y. Wu, J. Markmann, E.T. Lilleodden, Electro-chemo-mechanical coupling of nanoporous gold at the microscale. Appl. Phys. Lett. 115(25), 251602 (2019)

9. M. Tuller, R. Kulkarni, W. Fink, Segmentation of X-Ray CT Data of Porous Materials: A Review of Global and Locally Adaptive Algorithms, in Soil-Water-Root Processes: Advances in Tomography and Imaging (SSSA, Madison, 2013), pp. 157-182

10. W. Niblack, An Introduction to Digital Image Processing (Prentice Hall International, Englewood Cliffs, 1988)

11. J. Schindelin, I. Arganda-Carreras, E. Frise, V. Kaynig, M. Longair, T. Pietzsch, S. Preibisch, C. Rueden, S. Saalfeld, B. Schmid, J.-Y. Tinevez, D.J. White, V. Hartenstein, K. Eliceiri, P. Tomancak, A. Cardona, Fiji: an open-source platform for biologicalimage analysis. Nat. Methods 9(7), 676 (2012)

12. T. Prill, K. Schladitz, D. Jeulin, M. Faessel, C. Wieser, Morphological segmentation of FIB-SEM data of highly porous media. J. Microsc. 250(2), 77 (2013)

13. M. Röding, C. Fager, A. Olsson, C. von Corswant, E. Olsson, N. Lorén, Three-dimensional reconstruction of porous polymer films from FIB-SEM nanotomography data using random forests. J. Microsc. 281(1), 76 (2021)

14. The GIMP Development Team, GIMP (The GIMP Development Team, 2018). https://www.gimp.org 